Research Article

\title{
On Stability Analysis of Higher-Order Rational Difference Equation
}

\author{
Abdul Khaliq $\mathbb{D}^{1}{ }^{1}$ H. S. Alayachi, ${ }^{2}$ M. S. M. Noorani, ${ }^{2}$ and A. Q. Khan ${ }^{3}{ }^{3}$ \\ ${ }^{1}$ Department of Mathematics, Riphah Institute of Computing and Applied Sciences, Riphah International University, \\ Lahore Campus, Pakistan \\ ${ }^{2}$ School of Mathematical Sciences, Faculty of Science and Technology, University Kebangsaan Malaysia, Bangi Selangor, Malaysia \\ ${ }^{3}$ Departement of Mathematics, University of Azad Jammu and Kashmir, Muzaffarabad 13100, Pakistan
}

Correspondence should be addressed to Abdul Khaliq; khaliqsyed@gmail.com

Received 6 February 2020; Accepted 20 July 2020; Published 26 August 2020

Academic Editor: Rodica Luca

Copyright (c) 2020 Abdul Khaliq et al. This is an open access article distributed under the Creative Commons Attribution License, which permits unrestricted use, distribution, and reproduction in any medium, provided the original work is properly cited.

\begin{abstract}
In this paper, we study the equilibrium points, local asymptotic stability of equilibrium points, global behavior of equilibrium points, boundedness and periodicity of the rational recursive sequence $w_{n+1}=w_{n-p}\left(\alpha+\left(\beta w_{n} / \gamma w_{n}+\delta w_{n-r}\right)\right)$, where $\gamma w_{n} \neq-\delta w_{n-r}$ for $r \in(0, \infty), \alpha, \beta, \gamma, \delta \in(0, \infty)$, and $r>p \geq 0$. With initial values $w_{-p}, w_{-p+1}, \ldots, w_{-r}, w_{-r+1}, \ldots, w_{-1}$, and $w_{0}$ are positive real numbers. Some numerical examples are given to verify our theoretical results.
\end{abstract}

\section{Introduction}

It is very amusing to explore the nature of the solutions of a higher-order rational difference equation and to explain the local asymptotic stability of its equilibrium points. The inspection of some properties associated with these equations is a very enormous activity. Discrete dynamical systems or difference equations are diverse fields because various biological systems and models naturally lead to their study by means of a discrete variable. Applications of discrete dynamical systems and difference equations have appeared recently in many fields of science and technology. There is no doubt that the theory of difference equations will continue to play an important role in mathematics as a whole. Rational difference equations are a special form of nonlinear difference equations. Delay difference equations have rich dynamics to study. Due to adequate computational outcomes, discrete dynamical systems are awful lot better than allied structures in differential equations. Specifically, in case of nonoverlapping generations, difference equations are greater apposite to take a look at the behavior of population models $[1,2]$. Also, an epidemiological approach to insurgent population modeling is mentioned in $[3,4]$. The research of delay difference equations is a divergent field that involves most aspects of mathematics, including both applied and pure. Recently, there has been a symbolic development in the applications of difference equations. It is very interesting to investigate the behavior of solutions of a system of nonlinear difference equations and to discuss the local asymptotic stability of their equilibrium points. There are many papers in which systems and behavior of rational difference equations have been studied see [5-20]. Rational difference equations have been studied by several authors. There has been a great interest exclusively in the study of the attractiveness of the solution of such equations.

Here, we recall some basics and preliminaries that will be useful in our results and investigation.

\section{Preliminaries and Definitions}

Definition 1. Let $I$ be some interval of real numbers, and let

$$
F: I^{k+1} \longrightarrow I,
$$

be a continuously differentiable function. Then, for every set of initial conditions $S_{-k}, S_{-k+1}, \ldots, S_{0} \in I$, the difference equation 


$$
S_{n+1}=R\left(S_{n}, S_{n-1}, \ldots, S_{n-k}\right), \quad n=0,1, \ldots,
$$

has a unique solution $\left\{S_{n}\right\}_{n=-k}^{\infty}$.

Definition 2. A point $\bar{S} \in I$ is called an equilibrium point of equation (2) if

$$
\bar{S}=R(\bar{S}, \bar{S}, \ldots, \bar{S})
$$

That is, $S_{n}=\bar{S}$, for all $n \geq 0$, is a solution of equation (2), or equivalently, $\bar{S}$ is a fixed point of $R$.

Definition 3 (periodicity). A sequence $\left\{S_{n}\right\}_{n=-k}^{\infty}$ is said to be periodic with period $p$ if $S_{n+p}=S_{n}$ for all $n \geq-k$.

Definition 4 (Fibonacci sequence). The sequence $\left\{F_{m}\right\}_{m=1}^{\infty}=$ $\{1,2,3,5,8,13, \ldots\}$ i.e. $F_{m}=F_{m-1}+F_{m-2} \geq 0, F_{-2}=0$, $F_{-1}=1$ is called Fibonacci sequence.

Definition 5. Equation (2) is called permanent and bounded if there exist numbers $t$ and $T$ with $0<t<T<\infty$ such that for any initial values $S_{-k}, S_{-k+1}, \ldots, S_{-1}, S_{0} \in I$, there exists a positive integer $N$ which depends on these initial values such that $t \leq S_{n} \leq T$, for all $n \geq N$.

\section{Definition 6 (stability)}

(i) The equilibrium point $\bar{S} \in I$ of equation (2) is locally stable if for every $\varepsilon>0$, there exists $\delta>0$ such that for all $S_{-k}, S_{-k+1}, \ldots, S_{-1}, S_{0} \in I$ with

$$
\left|S_{-k}-\bar{S}\right|+\left|S_{-k+1}-\bar{S}\right|+\cdots+\left|S_{0}-\bar{S}\right|<\delta,
$$

we have

$$
\left|S_{n}-\bar{S}\right|<\varepsilon \text { for all } n \geq-k
$$

(ii) The equilibrium point $\bar{S}$ of equation (2) is locally asymptotically stable if $\bar{S}$ is a locally stable solution of equation (2) and there exists $\gamma>0$, such that for all $S_{-k}, S_{-k+1}, \ldots, S_{-1}, S_{0} \in K$ with

$$
\left|S_{-k}-\bar{S}\right|+\left|S_{-k+1}-\bar{S}\right|+\cdots+\left|S_{0}-\bar{S}\right|<\gamma,
$$

we have

$$
\lim _{n \longrightarrow \infty} S_{n}=\bar{S}
$$

(iii) The equilibrium point $\bar{S}$ of equation (2) is global attractor if for all $S_{-k}, S_{-k+1}, \ldots, S_{-1}, S_{0} \in I$, we have

$$
\lim _{n \longrightarrow \infty} S_{n}=\bar{S}
$$

(iv) The equilibrium point $\bar{S}$ of equation (2) is globally asymptotically stable if $\bar{S}$ is locally stable and $\bar{S}$ is also a global attractor of equation (2).

(v) The equilibrium point $\bar{S}$ of equation (2) is unstable if $\bar{S}$ is not locally stable.

Definition 7. The linearized equation of equation (2) about the equilibrium $\bar{S}$ is defined by the following equation:

$$
y_{n+1}=\sum_{i=0}^{3} \rho_{i} y_{n-i},
$$

where

$$
\begin{aligned}
\rho_{0} & =\frac{\partial F(\bar{S}, \bar{S}, \ldots, \bar{S})}{\partial S_{n}}, \\
\rho_{1} & =\frac{\partial F(\bar{S}, \bar{S}, \ldots, \bar{S})}{\partial S_{n-1}}, \\
\rho_{2} & =\frac{\partial F(\bar{S}, \bar{S}, \ldots, \bar{S})}{\partial S_{n-2}}, \\
\rho_{3} & =\frac{\partial F(\bar{S}, \bar{S}, \ldots, \bar{S})}{\partial S_{n-3}} .
\end{aligned}
$$

Lemma 1 (see[21]). Assume that $p_{i} \in R$ and $i \in\{1,2, \ldots, k\}$. Then,

$$
\sum_{i=1}^{k}\left|p_{i}\right|<1,
$$

is a sufficient condition for the asymptotic stability of the following difference equation:

$$
S_{n+k}+p_{1} S_{n+k-1}+\cdots+p_{k} S_{n}=0, \quad n=0,1,2, \ldots
$$

The following theorem will be useful for the proof of our results in this paper.

Theorem 1. Let $[\alpha, \beta]$ be an interval of real numbers and assume that $g:[\alpha, \beta]^{2} \longrightarrow[\alpha, \beta]$ is a continuous function, and consider the following equation:

$$
x_{n+1}=g\left(x_{n}, x_{n-1}\right), \quad n=0,1, \ldots,
$$

satisfying the following conditions:

(a) $g(x, y)$ is nondecreasing in $x \in[\alpha, \beta]$ for each fixed $y \in[\alpha, \beta]$, and $g(x, y)$ is nonincreasing in $y \in[\alpha, \beta]$ for each fixed $x \in[\alpha, \beta]$

(b) If $(m, M) \in[\alpha, \beta] \times[\alpha, \beta]$ is a solution of the systems

$$
\begin{aligned}
M & =g(M, m), \\
m & =g(m, M),
\end{aligned}
$$


then $m=M$ and equation (2) has a unique equilibrium $\bar{S} \in[\alpha, \beta]$ and every solution of equation (2) converges to $\bar{S}$.

Some published works include the following:

Ibrahim and El-Moneam [22] investigate the local and global stability of the following recursive sequence

$$
S_{n+1}=S_{n-p}\left(\alpha+\frac{b S_{n-q}}{c S_{n-q}+d S_{n-r}}\right),
$$

where $c S_{-q+k} \neq-d S_{-r+k}$ for $k=0,1, \ldots, \min (q, r)$ and $\alpha, b$, $c$, and $d$ are real numbers.

Aboutaleb et al. [23] studied the global attractiveness of the positive equilibrium of the following rational recursive equation:

$$
y_{m+1}=\frac{\alpha-\beta y_{m}}{\gamma+y_{m-1}}, \quad m=0,1,2, \ldots
$$

where the coefficients $\alpha, \beta$, and $\gamma$ are the nonnegative real numbers.

Several other researchers have studied the behavior of the solution of difference equations; for example, Devault et al. [24] investigated the global behavior of all positive solutions of the following equation:

$$
S_{n+1}=\frac{p+S_{n-k}}{q S_{n-k}} .
$$

Elabbasy et al. [25] studied the boundedness, global stability, and periodicity character and gave the solution of some special cases of the following difference equation:

$$
x_{n+1}=\frac{\alpha x_{n-l}+\beta x_{n-k}}{A x_{n-l}+B x_{n-k}} .
$$

Elabbasy [26] gave the solution of the following difference equation:

$$
S_{n+1}=\alpha S_{n}-\frac{b S_{n}}{c S_{n}-d S_{n-1}} .
$$

El-Moneam and Zayed [27] investigated the global stability and periodicity character and gave the solution of some special cases of the following difference equation:

$$
x_{n+1}=a x_{n-k}+\frac{b x_{n-k}}{c x_{n}+d x_{n-k}} .
$$

Zayed and El-Moneam [28] studied the global behavior of the following rational recursive sequence:

$$
x_{n+1}=\frac{\alpha_{0} x_{n}+\alpha_{1} x_{n-2}+\alpha_{2} x_{n-m}+\alpha_{3} x_{n-k}}{\beta_{0} x_{n}+\beta_{1} x_{n-2}+\beta_{2} x_{n-m}+\beta_{3} x_{n-k}} .
$$

Motivated by the above study, the main focus of this article is to discuss some qualitative behaviors of the solutions of the following rational recursive sequence:

$$
w_{n+1}=w_{n-p}\left(\alpha+\frac{\beta w_{n}}{\gamma w_{n}+\delta w_{n-r}}\right),
$$

where $\gamma w_{n} \neq-\delta w_{n-r}$ for $r \in(0, \infty), \alpha, \beta, \gamma, \delta \in(0, \infty)$, and $r>p \geq 0$. With initial values $w_{-p}, w_{-p+1}, \ldots w_{-r}, w_{-r+1}, \ldots w_{-1}$, and $w_{0}$ are the positive real numbers. Some numerical examples are given to verify our theoretical results.

\section{Local Stability of Equation (22)}

This section deals with the local stability character of the solutions of equation (22). Equation (22) has a unique equilibrium point, and it is given from

$$
\begin{aligned}
\bar{w} & =\bar{w}\left(\alpha+\frac{\beta \bar{w}}{\gamma \bar{w}+\delta \bar{w}}\right), \\
\text { or } \bar{w}^{2}(1-\alpha)(\gamma+\delta) & =\beta \bar{w}^{2},
\end{aligned}
$$

if

$$
(1-\alpha)(\gamma+\delta) \neq \beta
$$

Then, the unique equilibrium point is $\bar{w}=0$.

Let $f:(0, \infty)^{3} \longrightarrow(0, \infty)$ be a function defined by

$$
f(s, u, v)=\alpha s+\frac{\beta s u}{\gamma u+\delta v}
$$

Therefore, it follows that

$$
\begin{aligned}
& f_{s}=\alpha+\frac{\beta u}{\gamma u+\delta v}, \\
& f_{u}=\frac{\delta \beta v s}{(\gamma u+\delta v)^{2}}, \\
& f_{v}=\frac{-\beta s u}{(\gamma u+\delta v)^{2}} .
\end{aligned}
$$

We see that

$$
\begin{aligned}
& f_{s}(\bar{w}, \bar{w}, \bar{w})=\alpha+\frac{\beta}{\gamma+\delta}=-a_{2}, \\
& f_{u}(\bar{w}, \bar{w}, \bar{w})=\frac{\delta \beta}{(\gamma+\delta)^{2}}=-a_{1}, \\
& f_{v}(\bar{w}, \bar{w}, \bar{w})=\frac{-\beta \delta}{(\gamma+\delta)^{2}}=-a_{0} .
\end{aligned}
$$

The linearized equation of equation (22) about $\bar{w}$ is

$$
T_{n+1}=a_{0} T_{n}+a_{1} T_{n-p}+a_{2} T_{n-r}
$$

The characteristic equation of equation (28) is

$$
\lambda^{n+1}=a_{0} \lambda^{n}+a_{1} \lambda^{n-p}+a_{2} \lambda^{n-r} .
$$

Theorem 2. Assume that

$$
(\gamma+\delta)^{2}(1-\alpha)>\beta(\gamma+3 \delta)
$$

Then, the equilibrium point of equation (22) is locally asymptotically stable.

Proof. It follows by Lemma 7 that equation (28) is asymptotically stable if 


$$
\begin{array}{r}
\left|\alpha+\frac{\beta}{\gamma+\delta}\right|+\left|\frac{\delta \beta}{(\gamma+\delta)^{2}}\right|+\left|\frac{-\beta \delta}{(\gamma+\delta)^{2}}\right|<1, \\
\text { or } \alpha+\frac{\beta}{\gamma+\delta}+\frac{2 \delta \beta}{(\gamma+\delta)^{2}}<1,
\end{array}
$$

and so

$$
\frac{\beta \gamma+3 \beta \delta}{(\gamma+\delta)^{2}}<(1-\alpha)
$$

Hence,

$$
(\gamma+\delta)^{2}(1-\alpha)>\beta(\gamma+3 \delta)
$$

\section{Boundedness of the Solutions}

In this section, we show the boundedness of the positive solutions of equation (22).

Theorem 3. Every solution of equation (22) is bounded from above by

$$
M=\max \left\{w_{-(p+1)}, w_{-p}, \ldots, w_{-2}, w_{-1}, w_{0}\right\},
$$

if

$$
\alpha+\frac{\beta}{\gamma}<1
$$

Proof. Let $\left\{F_{n}\right\}_{n=-p}^{\infty}$ be a solution of equation (22). It follows from equation (22) that

$$
w_{n+1}=\alpha w_{n-p}+\frac{\beta w_{n} w_{n-p}}{\gamma w_{n}+\delta w_{n-r}} \leq \alpha w_{n-p}+\frac{\beta w_{n} w_{n-p}}{\gamma w_{n}}=\left(\alpha+\frac{\beta}{\gamma}\right) w_{n-p} .
$$

Then, for all $n \geq N$,

$$
w_{n+1} \leq w_{n-p}, \quad \forall n \geq 0 .
$$

Then, all the subsequences $\left\{w_{(p+1) n+j}\right\}_{n=0}^{\infty}, i=0,1,2, \ldots$, $p$, are decreasing and so are bounded from above by

$$
M=\max \left\{w_{-(p+1)}, w_{-p}, \ldots, w_{-2}, w_{-1}, w_{0}\right\} .
$$

Theorem 4. If $\alpha>1$, then each solution of equation (22) will be unbounded.

Proof. Let $\left\{w_{n}\right\}_{n=-p}^{\infty}$ be a solution of equation (22). Then, from equation (22), we see that

$$
w_{n+1}=\alpha w_{n-p}+\frac{\beta w_{n} w_{n-p}}{\gamma w_{n}+\delta w_{n-r}}>\alpha w_{n-p}, \quad \forall n \geq 1 .
$$

From above, the right-hand side can be written as follows:

$$
T_{n+1}=\alpha T_{n-p} \Longrightarrow T_{n}=\alpha^{n} T_{0}
$$

and this equation is unstable because $\alpha>1$ and $\lim _{n \rightarrow \infty} T_{n}=\infty$.

Then, by using the ratio test, $\left\{w_{n}\right\}_{n=-p}^{\infty}$ is unbounded from above.

\section{Periodic Solutions of Equation (22)}

In this section, we satisfy the periodic solutions of equation (22).

Theorem 5. Equation (22) has no positive solutions of prime period two solution $\forall \alpha, \beta, \gamma, \delta \in(0, \infty)$ in the following case, if $p$ is even and $r$ is odd.

Proof. Assume that there exists two distinct positive real numbers $\phi, \varphi \in(0, \infty)$ such that $\phi, \varphi, \phi, \varphi, \phi, \varphi, \ldots$ is prime period two solution of equation (22). We have

$$
\begin{aligned}
& \phi=\varphi\left(\alpha+\frac{\beta \varphi}{\gamma \varphi+\delta \phi}\right), \\
& \varphi=\phi\left(\alpha+\frac{\beta \phi}{\gamma \phi+\delta \varphi}\right) .
\end{aligned}
$$

By simplifying equations (41) and (42), we obtain

$$
(\phi-\varphi \alpha)(\gamma \varphi-\delta \phi)=\beta \varphi^{2}(\varphi-\phi \alpha)(\gamma \phi-\delta \varphi)=\beta \phi^{2} .
$$

By subtracting, we deduce that

$$
\begin{aligned}
& (\phi-\varphi \alpha)(\gamma \varphi-\delta \phi)-(\varphi-\phi \alpha)(\gamma \phi-\delta \varphi)=\beta \varphi^{2} \\
& \quad-\beta \phi^{2}(\delta+\beta+\alpha \gamma) \phi^{2}=(\beta+\alpha \gamma+\delta) \varphi^{2} \Longrightarrow \phi^{2}-\varphi^{2}=0 .
\end{aligned}
$$

This implies that equation (22) has no positive solutions of prime period two.

Theorem 6. Equation (22) has positive period two solution $\forall \alpha, \beta, \gamma, \delta \in(0, \infty)$ in the following cases:

(i) $(\gamma+\delta)(1+\alpha)+\beta=0(p$ and $r$ are even $)$

(ii) $\alpha=1$ ( $p$ and $r$ are odd $)$

(iii) $(\gamma+\delta)(1-\alpha)-\beta=0$ ( $p$ is odd; $r$ is even $)$

Proof. We will prove case (i) and the other cases (ii) and (iii) are obtained by the same way. Assume that there exist two distinct positive real numbers $\phi, \varphi \in(0, \infty)$ such that $\phi, \varphi$, $\phi, \varphi, \phi, \varphi, \ldots$ is prime period two solution of equation (22):

$$
\begin{aligned}
& \phi=\varphi\left(\alpha+\frac{\beta \varphi}{\gamma \varphi+\delta \varphi}\right), \\
& \varphi=\phi\left(\alpha+\frac{\beta \phi}{\gamma \phi+\delta \phi}\right) .
\end{aligned}
$$

From equation (45) and equation (46), we obtain

$$
(\phi-\varphi \alpha)(\gamma+\delta)=\beta \varphi(\varphi-\phi \alpha)(\gamma+\delta)=\beta \phi,
$$

By solving these equations, we obtain 


$$
(\phi-\varphi)(\gamma+\delta)(1+\alpha)+\beta=0 .
$$

Since $\alpha, \beta, \gamma$, and $\delta$ are nonzero positive real numbers, and $\phi \neq \varphi$.

This implies that

$$
(\gamma+\delta)(1+\alpha)+\beta=0
$$

\section{Global Stability}

We will study the global asymptotic stability of the positive solutions of equation (22) in this section.

Theorem 7. The equilibrium point $\bar{w}$ of equation (22) is a global attractor if

$$
\beta \neq(1-\alpha) \gamma
$$

Proof. Let $a$ and $b$ are real numbers and assume that $f:(a, b)^{3} \longrightarrow(a, b)$ be function defined by $f(s, u, v)=\alpha s+(\beta s u / \gamma u+\delta v)$. Thus, we see that the function $f(s, u, v)$ is increasing in $s$ and $u$ and is decreasing in $v$. Let $(g, G)$ is a solution of the system $G=f(G, G, g)$ and $g=f(g, g, G)$. Then, from (22), we see that

$$
\begin{gathered}
G=\alpha G+\frac{\beta G^{2}}{\gamma G+\delta g}, \\
g=\alpha g+\frac{\beta g^{2}}{\gamma g+\delta G},
\end{gathered}
$$

$$
g(1-\alpha)=\frac{\beta g^{2}}{\gamma g+\delta G} \text {. }
$$

Then,

$$
\begin{aligned}
& \gamma(1-\alpha) G^{2}+\delta(1-\alpha) G g=G^{2} \beta, \\
& \gamma(1-\alpha) g^{2}+\delta(1-\alpha) G g=g^{2} \beta .
\end{aligned}
$$

By subtracting these equations, we obtain

$$
\gamma(1-\alpha)\left(G^{2}-g^{2}\right)=\beta\left(G^{2}-g^{2}\right), \quad \gamma(1-\alpha) \neq \beta .
$$

Thus,

$$
G=g
$$

It follows that the equilibrium point $\bar{w}$ of equation (22) is a global attractor.

\section{Applications}

In this section, we will discuss the solution of some special cases of equation (22).

Case 1. When $\alpha=0, p=r=0$. In this case, we have the following special type of difference equation:

$$
w_{n+1}=\frac{\beta w_{n}^{2}}{\gamma w_{n}+\delta w_{n}}=w_{n}\left(\frac{\beta}{\gamma+\delta}\right) .
$$

Theorem 8. The general solution of equation (56) is

$$
w_{n}=\frac{a \beta^{n}}{(\gamma+\delta)^{n}},
$$

where

$$
w_{0}=a
$$

Proof. Proof is obtained by induction, and it is easy to do.

Case 2. When $\alpha=1$ and $\beta=\gamma=\delta=1, p=3$ and $r=4$. In this case, we have the following special case of difference equation:

$$
w_{n+1}=w_{n-3}+\frac{w_{n} w_{n-3}}{w_{n}+w_{n-4}}
$$

where $w_{-4}, w_{-3}, w_{-2}, w_{-1}$, and $w_{0}$ are the arbitrary real numbers.

Theorem 9. Let $\left\{w_{n}\right\}_{n=-4}^{\infty}$ be a solution of equation (59). Then, for $n=0,1,2, \ldots$,

$$
\begin{gathered}
w_{4 n}=e \prod_{i=0}^{n-1}\left(\frac{F_{8 i+8} a+F_{8 i+9} e}{F_{8 i+7} a+F_{8 i+8} e}\right), \\
w_{4 n-1}=d \prod_{i=0}^{n-1}\left(\frac{F_{8 i+6} a+F_{8 i+7} e}{F_{8 i+5} a+F_{8 i+6} e}\right), \\
w_{4 n-2}=c \prod_{i=0}^{n-1}\left(\frac{F_{8 i+4} a+F_{8 i+5} e}{F_{8 i+3} a+F_{8 i+4} e}\right),
\end{gathered}
$$

where $w_{-4}=a, w_{-3}=b, w_{-2}=c, w_{-1}=d, w_{0}=e$, and $\left\{F_{m}\right\}_{m=0}^{\infty}=\{0,1,1,2,3,5,8,13,21, \ldots$,$\} .$

Proof. For $n=0$, the result holds. Now, suppose that $n>0$ and that our assumption holds for $n-1$ and $n-2$ : 


$$
\begin{aligned}
& w_{4 n-4}=e \prod_{i=0}^{n-2}\left(\frac{F_{8 i+8} a+F_{8 i+9} e}{F_{8 i+7} a+F_{8 i+8} e}\right) \\
& w_{4 n-5}=d \prod_{i=0}^{n-2}\left(\frac{F_{8 i+6} a+F_{8 i+7} e}{F_{8 i+5} a+F_{8 i+6} e}\right) \\
& w_{4 n-6}=c \prod_{i=0}^{n-2}\left(\frac{F_{8 i+4} a+F_{8 i+5} e}{F_{8 i+3} a+F_{8 i+4} e}\right) \\
& w_{4 n-7}=b \prod_{i=0}^{n-2}\left(\frac{F_{8 i+2} a+F_{8 i+3} e}{F_{8 i+1} a+F_{8 i+2} e}\right) \\
& w_{4 n-8}=e \prod_{i=0}^{n-3}\left(\frac{F_{8 i+8} a+F_{8 i+9} e}{F_{8 i+7} a+F_{8 i+8} e}\right)
\end{aligned}
$$

Now, it follows from (59) that

$$
\begin{aligned}
& w_{4 n}=w_{n-4}-\frac{w_{4 n-1} w_{4 n-4}}{w_{4 n-1}-w_{4 n-5}} \\
& =e \prod_{i=0}^{n-2}\left(\frac{F_{8 i+8} a+F_{8 i+9} e}{F_{8 i+7} a+F_{8 i+8} e}\right) \\
& -\frac{d \prod_{i=0}^{n-1}\left(F_{8 i+6} a+F_{8 i+7} e / F_{8 i+5} a+F_{8 i+6} e\right) e \prod_{i=0}^{n-2}\left(F_{8 i+8} a+F_{8 i+9} e / F_{8 i+7} a+F_{8 i+8} e\right)}{d \prod_{i=0}^{n-1}\left(F_{8 i+6} a+F_{8 i+7} e / F_{8 i+5} a+F_{8 i+6} e\right)-d \prod_{i=0}^{n-2}\left(F_{8 i+6} a+F_{8 i+7} e / F_{8 i+5} a+F_{8 i+6} e\right)} \\
& =e \prod_{i=0}^{n-2}\left(\frac{F_{8 i+8} a+F_{8 i+9} e}{F_{8 i+7} a+F_{8 i+8} e}\right)\left[1-\frac{d \prod_{i=0}^{n-1}\left(F_{8 i+6} a+F_{8 i+7} e / F_{8 i+5} a+F_{8 i+6} e\right)}{d \prod_{i=0}^{n-1}\left(F_{8 i+6} a+F_{8 i+7} e / F_{8 i+5} a+F_{8 i+6} e\right)-d \prod_{i=0}^{n-2}\left(F_{8 i+6} a+F_{8 i+7} e / F_{8 i+5} a+F_{8 i+6} e\right)}\right] \\
& =e \prod_{i=0}^{n-2}\left(\frac{F_{8 i+8} a+F_{8 i+9} e}{F_{8 i+7} a+F_{8 i+8} e}\right)\left[1-\frac{d \prod_{i=0}^{n-1}\left(F_{8 i+6} a+F_{8 i+7} e / F_{8 i+5} a+F_{8 i+6} e\right)}{d \prod_{i=0}^{n-1}\left(F_{8 i+6} a+F_{8 i+7} e / F_{8 i+5} a+F_{8 i+6} e\right)\left[\left(F_{8 n-2} a+F_{8 n-1} e / F_{8 n-3} a+F_{8 n-2} e\right)-1\right]}\right] \\
& =e \prod_{i=0}^{n-2}\left(\frac{F_{8 i+8} a+F_{8 i+9} e}{F_{8 i+7} a+F_{8 i+8} e}\right)\left[1-\left(\frac{F_{8 n-2} a+F_{8 n-1} e / F_{8 n-3} a+F_{8 n-2} e}{F_{8 n-2} a+F_{8 n-1} e-F_{8 n-3} a-F_{8 n-2} e / F_{8 n-3} a+F_{8 n-2} e}\right)\right] \\
& =e \prod_{i=0}^{n-2}\left(\frac{F_{8 i+8} a+F_{8 i+9} e}{F_{8 i+7} a+F_{8 i+8} e}\right)\left[1-\frac{F_{8 n-2} a+F_{8 n-1} e}{F_{8 n-1} a+F_{8 n} e}\right] \\
& =e \prod_{i=0}^{n-2}\left(\frac{F_{8 i+8} a+F_{8 i+9} e}{F_{8 i+7} a+F_{8 i+8} e}\right)\left[\frac{F_{8 n} a+F_{8 n+1} e}{F_{8 n-1} a+F_{8 n} e}\right] .
\end{aligned}
$$

Thus, we have

$$
w_{4 n}=e \prod_{i=0}^{n-1}\left(\frac{F_{8 i+8} a+F_{8 i+9} e}{F_{8 i+7} a+F_{8 i+8} e}\right) .
$$

Hence, the proof is completed.

Similarly, we can prove other relations.

Case 3. When $\alpha=0$ and $\beta=\gamma=\delta=1, p=r=3$. In this case, we have the following special case of difference equation:

$$
w_{n+1}=\frac{w_{n} w_{n-3}}{w_{n}+w_{n-3}}
$$

The following theorem gives the solution of equation (64).

Theorem 10. Let $\left\{w_{n}\right\}_{n=-p}^{\infty}$ be a solution of equation (64). Then, the solution for equation (64) is given by

$$
w_{n}=\frac{w_{0} w_{-1} w_{-2} w_{-3}}{F_{n-2} w_{0} w_{-1} w_{-3}+F_{n-1} w_{0} w_{-2} w_{-3}+F_{n} w_{-1} w_{-3} w_{-2}+F_{n+1} w_{0} w_{-1} w_{-2}} \text {, }
$$


where $F_{n}=F_{n-1}+F_{n-3}, n \geq 4, F_{0}=0$, and $F_{1}=F_{2}=F_{3}=1$ Proof. For $n=1$, and initial values $w_{-3}=d, w_{-2}=c, w_{-1}=b$, and $w_{0}=a$ are the arbitrary positive real numbers.

$$
\begin{aligned}
w_{1} & =\frac{w_{0} w_{-3}}{w_{0}+w_{-3}}=\frac{w_{0} w_{-1} w_{-2} w_{-3}}{w_{0} w_{-1} w_{-2}+w_{-1} w_{-2} w_{-3}} \\
& =\frac{w_{0} w_{-1} w_{-2} w_{-3}}{\left(F_{0}\right) w_{0} w_{-1} w_{-3}+\left(F_{1}\right) w_{0} w_{-2} w_{-3}+\left(F_{2}\right) w_{-1} w_{-3} w_{-2}+\left(F_{3}\right) w_{0} w_{-1} w_{-2}}
\end{aligned}
$$

Hence, the relation holds true. Now, suppose that relation (64) holds for $n=k$.
That is,

$$
w_{k}=\frac{w_{0} w_{-1} w_{-2} w_{-3}}{\left(F_{k-2}\right) w_{0} w_{-1} w_{-3}+\left(F_{k-1}\right) w_{0} w_{-2} w_{-3}+\left(F_{k}\right) w_{-1} w_{-3} w_{-2}+\left(F_{k+1}\right) w_{0} w_{-1} w_{-2}}
$$

We now want to show that relation (64) holds true for $n=k+1$ :

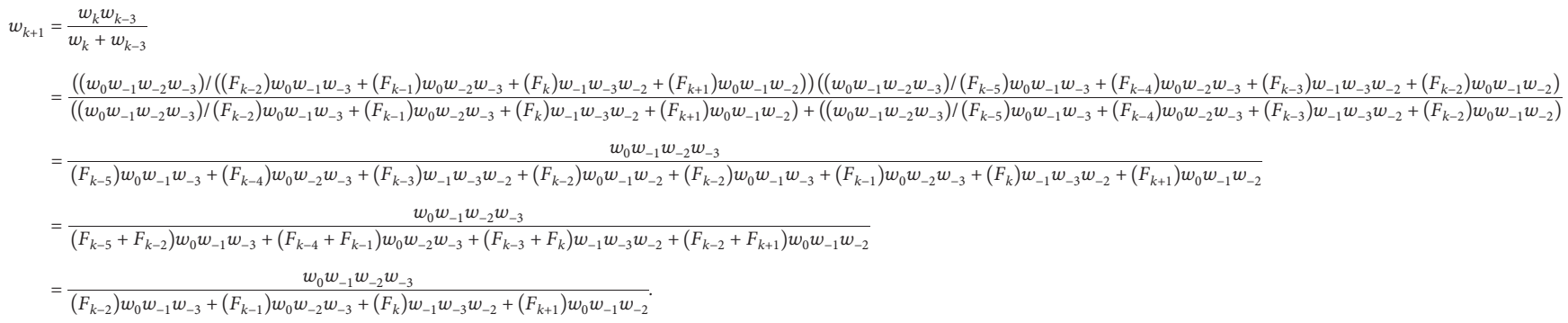

Case 4. When $\alpha=1$ and $\beta=\gamma=\delta=1, p=2$ and $r=3$. In this case, we have the following special case of difference equation:

$$
w_{n+1}=w_{n-2}+\frac{w_{n} w_{n-2}}{w_{n}+w_{n-3}},
$$

where $w_{-3}, w_{-2}, w_{-1}$, and $w_{0}$ are the arbitrary real numbers.

Theorem 11. Let $\left\{w_{n}\right\}_{n=-3}^{\infty}$ be a solution of equation (69). Then, for $n=0,1,2, \ldots$,

$$
\begin{aligned}
w_{3 n} & =d \prod_{i=0}^{n-1}\left(\frac{F_{6 i+6} a+F_{6 i+7} d}{F_{6 i+5} a+F_{6 i+6} d}\right), \\
w_{3 n+2} & =c \prod_{i=0}^{n-1}\left(\frac{F_{6 i+4} a+F_{6 i+5} d}{F_{6 i+3} a+F_{6 i+4} d}\right), \\
w_{3 n+1} & =b \prod_{i=0}^{n-1}\left(\frac{F_{6 i+2} a+F_{6 i+3} d}{F_{6 i+1} a+F_{6 i+2} d}\right),
\end{aligned}
$$

where $\quad w_{-3}=d, w_{-2}=c, w_{-1}=b, \quad w_{0}=a, \quad$ and $\left\{F_{m}\right\}_{m=0}^{\infty}=\{0,1,1,2,3,5,8,13,21, \ldots$,$\} .$

Proof. For $n=0$, the result holds. Now, suppose that $n>0$ and that our assumption holds for $n-1$ and $n-2$ :

$$
\begin{aligned}
& w_{3 n-3}=d \prod_{i=0}^{n-2}\left(\frac{F_{6 i+6} a+F_{6 i+7} d}{F_{6 i+5} a+F_{6 i+6} d}\right), \\
& w_{3 n-1}=c \prod_{i=0}^{n-2}\left(\frac{F_{6 i+4} a+F_{6 i+5} d}{F_{6 i+3} a+F_{6 i+4} d}\right), \\
& w_{3 n-2}=b \prod_{i=0}^{n-2}\left(\frac{F_{6 i+2} a+F_{6 i+3} d}{F_{6 i+1} a+F_{6 i+2} d}\right) .
\end{aligned}
$$




$$
\begin{aligned}
& w_{3 n}=w_{3 n-3}-\frac{w_{3 n+1} w_{3 n-3}}{w_{3 n+1}-w_{3 n-2}} \\
& =d \prod_{i=0}^{n-2}\left(\frac{F_{6 i+6} a+F_{6 i+7} d}{F_{6 i+5} a+F_{6 i+6} d}\right)-\frac{b \prod_{i=0}^{n-1}\left(F_{6 i+2} a+F_{6 i+3} d / F_{6 i+1} a+F_{6 i+2} d\right) d \prod_{i=0}^{n-2}\left(F_{6 i+6} a+F_{6 i+7} d / F_{6 i+5} a+F_{6 i+6} d\right)}{b \prod_{i=0}^{n-1}\left(F_{6 i+2} a+F_{6 i+3} d / F_{6 i+1} a+F_{6 i+2} d\right)-b \prod_{i=0}^{n-2}\left(F_{6 i+2} a+F_{6 i+3} d / F_{6 i+1} a+F_{6 i+2} d\right)} \\
& =d \prod_{i=0}^{n-2}\left(\frac{F_{6 i+6} a+F_{6 i+7} d}{F_{6 i+5} a+F_{6 i+6} d}\right)\left[1-\frac{b \prod_{i=0}^{n-1}\left(F_{6 i+2} a+F_{6 i+3} d / F_{6 i+1} a+F_{6 i+2} d\right)}{b \prod_{i=0}^{n-1}\left(F_{6 i+2} a+F_{6 i+3} d / F_{6 i+1} a+F_{6 i+2} d\right)-b \prod_{i=0}^{n-2}\left(F_{6 i+2} a+F_{6 i+3} d / F_{6 i+1} a+F_{6 i+2} d\right)}\right] \\
& =d \prod_{i=0}^{n-2}\left(\frac{F_{6 i+6} a+F_{6 i+7} d}{F_{6 i+5} a+F_{6 i+6} d}\right)\left[1-\frac{b \prod_{i=0}^{n-1}\left(F_{6 i+2} a+F_{6 i+3} d / F_{6 i+1} a+F_{6 i+2} d\right)}{b \prod_{i=0}^{n-2}\left(F_{6 i+2} a+F_{6 i+3} d / F_{6 i+1} a+F_{6 i+2} d\right)\left[\left(F_{6 i n-4} a+F_{6 n-3} d / F_{6 n-5} a+F_{6 i n-4} d\right)-1\right]}\right] \\
& =d \prod_{i=0}^{n-2}\left(\frac{F_{6 i+6} a+F_{6 i+7} d}{F_{6 i+5} a+F_{6 i+6} d}\right)\left[1-\frac{F_{6 i n-4} a+F_{6 n-3} d / F_{6 n-5} a+F_{6 i n-4} d}{F_{6 i n-4} a+F_{6 n-3} d-F_{6 n-5} a-F_{6 i n-4} d / F_{6 n-5} a+F_{6 i n-4} d}\right] \\
& =d \prod_{i=0}^{n-2}\left(\frac{F_{6 i+6} a+F_{6 i+7} d}{F_{6 i+5} a+F_{6 i+6} d}\right)\left[1-\frac{F_{6 n-4} a+F_{6 n-3} d}{F_{6 n-3} a+F_{6 n-2} d}\right] \\
& =d \prod_{i=0}^{n-2}\left(\frac{F_{6 i+6} a+F_{6 i+7} d}{F_{6 i+5} a+F_{6 i+6} d}\right)\left[\frac{F_{6 n-3} a-F_{6 n-2} d-F_{6 n-4} a+F_{6 n-3} d}{F_{6 n-3} a+F_{6 n-2} d}\right] \\
& =d \prod_{i=0}^{n-2}\left(\frac{F_{6 i+6} a+F_{6 i+7} d}{F_{6 i+5} a+F_{6 i+6} d}\right)\left[\frac{F_{6 n-2} a+F_{6 n-1} d}{F_{6 n-3} a+F_{6 n-2} d}\right], \\
& w_{3 n}=d \prod_{i=0}^{n-1}\left(\frac{F_{6 i+6} a+F_{6 i+7} d}{F_{6 i+5} a+F_{6 i+6} d}\right) .
\end{aligned}
$$

Hence, the proof is complete.

By using the same way, we can prove other relations.

\section{Numerical Examples}

This section discusses some numerical results of our previous results.

Example 1. Figure 1 shows the behavior of the solution bounded of equation (56) when we take

$$
\begin{aligned}
w_{0} & =0.1, \\
\alpha & =0, \\
\beta & =\gamma=\delta=1, \\
\alpha & =0, \\
p & =r=0 .
\end{aligned}
$$

Example 2. Figure 2 shows the behavior of solution of equation (59) when we take

$$
\begin{aligned}
w_{-4} & =1, \\
w_{-3} & =2, \\
w_{-2} & =3, \\
w_{-1} & =4, \\
w_{0} & =5, \\
\alpha & =1, \\
\beta & =\gamma=\delta=1, \\
p & =3, \\
r & =4 .
\end{aligned}
$$

Example 3. Figure 3 shows that the solution of equation (64) is globally asymptotically stable when we take 


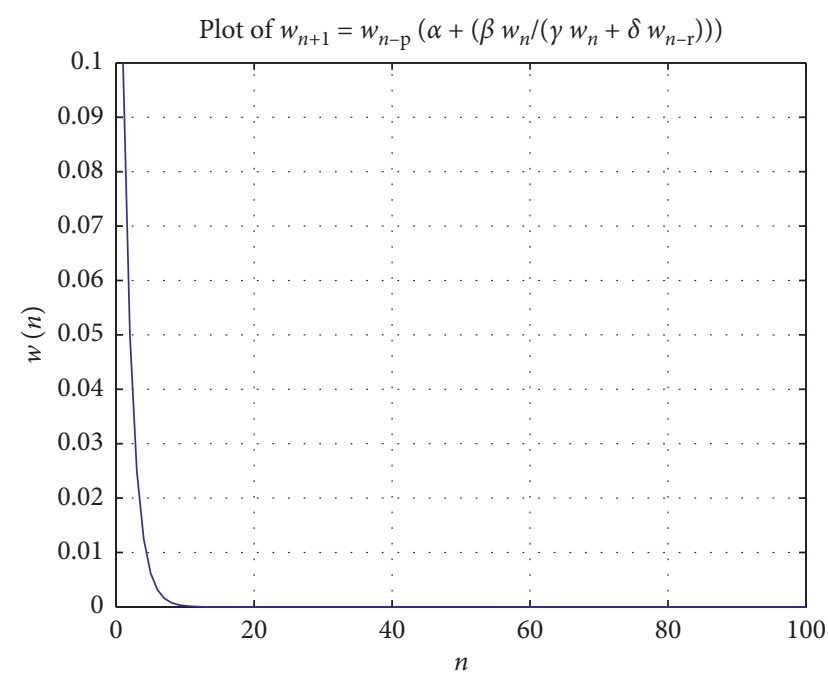

Figure 1: Bounded solution of equation (56).

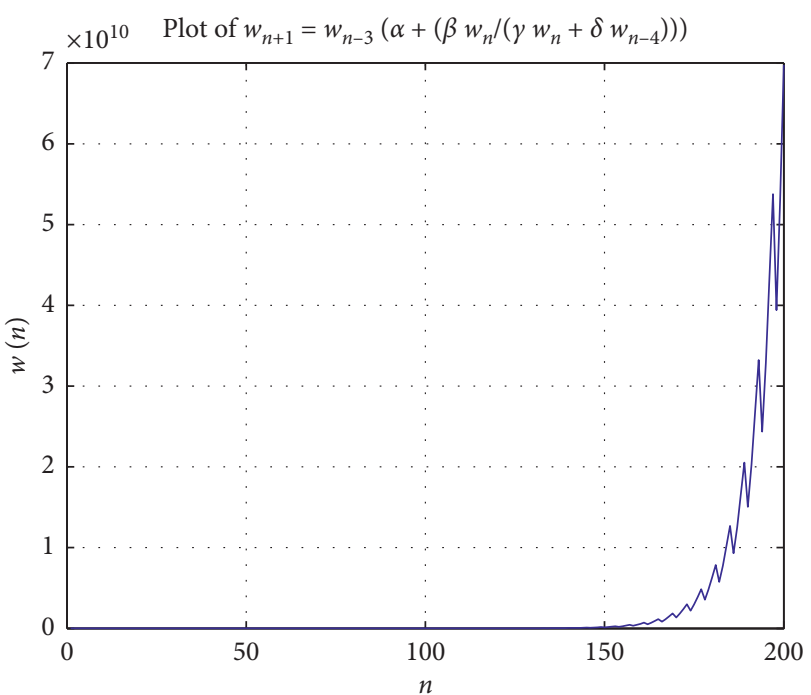

FIGURE 2: Unbounded solution of equation (59).

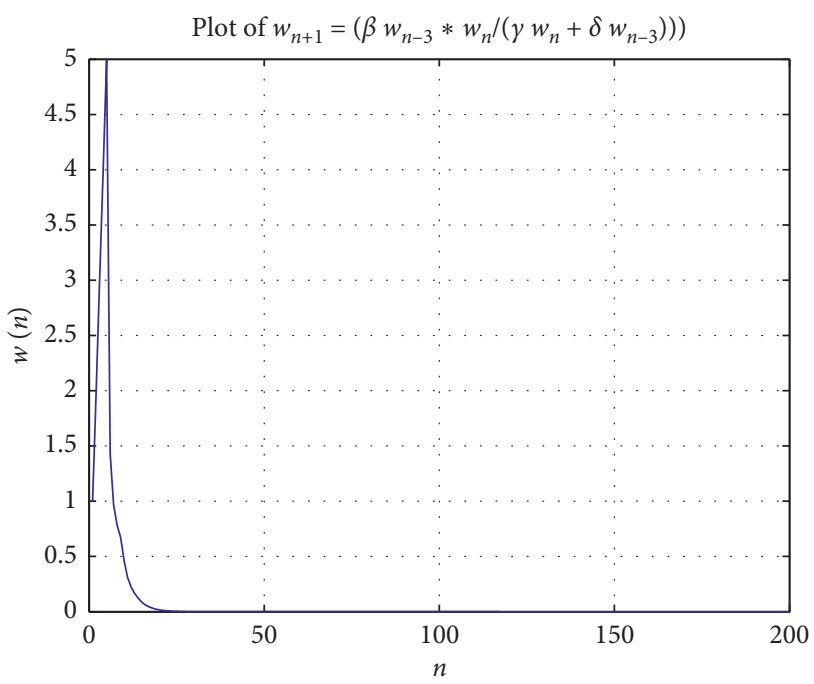

Figure 3: Global asymptotic stability of equation (64).

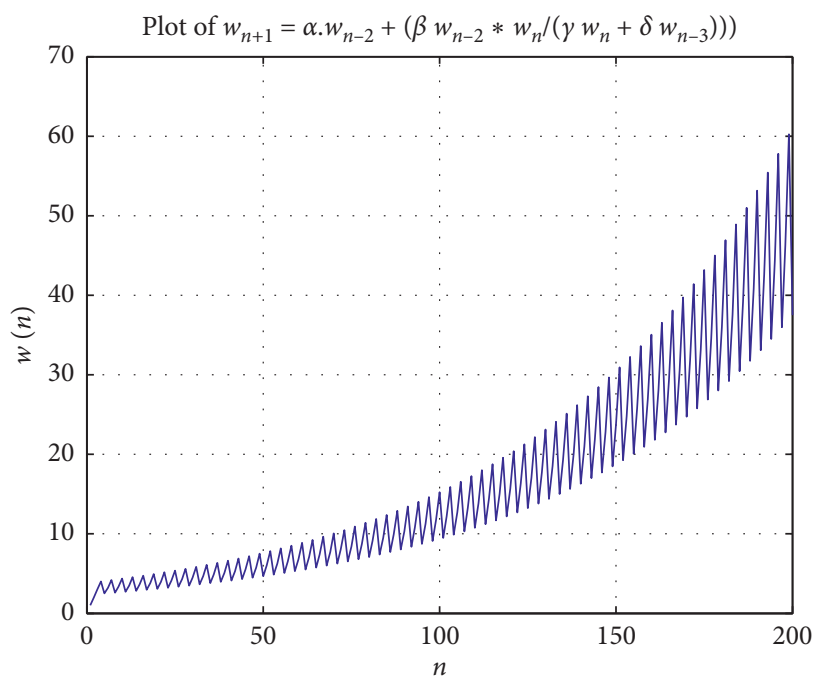

FIGURE 4: Equation (69) has no positive prime period two solution.

$$
\begin{aligned}
w_{-3} & =1, \\
w_{-2} & =2, \\
w_{-1} & =3, \\
w_{0} & =4, \\
\alpha & =0, \\
\beta & =\gamma=\delta=1, \\
p & =r=3 .
\end{aligned}
$$

Example 4. Figure 4 shows the solution of equation (69) has no prime period two solution if we take

$$
\begin{aligned}
w_{-3} & =0.75, \\
w_{-2} & =20, \\
w_{-1} & =30, \\
w_{0} & =40, \\
\alpha & =1, \\
\beta & =\gamma=\delta=1, \\
p & =2, \\
r & =3 .
\end{aligned}
$$

\section{Concluding Remarks}

In the literature, several articles are related to qualitative behavior of rational difference equations. It is a very interesting mathematical problem to study the dynamics of such equations because these are closely related to models in population dynamics and biological sciences. We have investigated the existence and uniqueness of positive equilibrium points, and boundedness and persistence of positive solutions are proved. Moreover, we have shown that they are locally as well as globally asymptotically stable. The main objective of dynamical systems theory is to predict the global 
behavior of a system based on the knowledge of its present state. We have taken some special cases as applications of equation (22) in Section 7 and found the closed form of the solutions. Finally, some illustrative examples are provided to support our theoretical discussion.

\section{Data Availability}

All the data utilized are included in this article and their sources are cited accordingly.

\section{Conflicts of Interest}

The authors declare that they have no conflicts of interest.

\section{References}

[1] R. P. Agarwal, Difference Equations and Inequalities, Dekker, New York, NY, USA, 2nd edition, 2000.

[2] S. Elaydi, An Introduction to Difference Equations, Springer, Berlin, Germany, 2nd edition, 2005.

[3] M. A. Khan and A. Atangana, "Modeling the dynamics of novel coronavirus (2019-nCov) with fractional derivative," Alexandria Engineering Journal, vol. 59, pp. 1117-1134, 2020.

[4] O. Kolebaje, O. Popoola, M. A. Khan, and O. Oyewande, "An epidemiological approach to insurgent population modeling with the Atangana-Baleanu fractional derivative," Chaos, Solitons \& Fractals, vol. 139, Article ID 109970, 2020.

[5] E. M. Elsayed, "On the global attractivity and the periodic character of a recursive sequence," Opuscula Mathematica, vol. 30, no. 4, pp. 431-446, 2010.

[6] M. Saleh and S. Abu-Baha, "Dynamics of a higher order rational difference equation," Applied Mathematics and Computation, vol. 181, no. 1, pp. 84-102, 2006.

[7] E. M. E. Zayad and M. A. El-Moneam, "On the rational recursive sequance $x_{n+1}=D+\alpha x_{n}+\beta x_{n-1}+\gamma x_{n-2} / A x_{n}+$ $B x_{n-1}+C x_{n-2}$." Communications on Applied Nonlinear Analysis, vol. 12, pp. 15-28, 2005.

[8] E. M. E. Zayad and M. A. El-Moneam, "On the global attractivity of two nonlinear difference equation," Journal of Mathematical Sciences, vol. 177, pp. 487-499, 2011.

[9] N. C. Battaloglu and T. M. Yalcinkaya, "ARS Combination," Journal of Mathematical Sciences, vol. 977, pp. 281-288, 2010.

[10] Q. Din, "On a system of rational difference equation," Demonstratio Mathematica, vol. 47, no. 2.

[11] S. E. Das and Bayram, "On a system of rational difference equation," World Applied Sciences Journal, vol. 10, no. 11, pp. 1306-1312, 2010.

[12] E. Elabbasy, H. A. El Metwally, and E. M. Elsayed, "Global behaviour of the solution of difference equation," Advances in Difference Equations, vol. 2011, no. 1, 2011.

[13] E. M. Elsayed, "Solutions of rational difference systems of order two," Mathematical and Computer Modelling, vol. 55, no. 3-4, pp. 378-384, 2012.

[14] M. A. El-Moneam, "On the dynamics of the higher order nonlinear rational difference equations," Mathematical Sciences Letters, vol. 3, no. 2, pp. 121-129, 2014.

[15] M. A. El-Moneam and E. M. E. Zayed, "Dynamics of the rational difference equation $x_{n+1}=A x_{n}+B x_{n-k}+C x_{n-1}+$ $\left(b x_{n} x_{n-k} x_{n-1} / d x_{n-k}-e x_{n-1}\right) . " D C D I S$ Series A: Mathematical Analysis, vol. 21, pp. 317-331, 2014.
[16] E. A. L. Grove and G. M. Teixeira, "Existence and behavior of solutions of a rational system," Communications on Pure \& Applied Analysis, vol. 8, pp. 1-25, 2001.

[17] T. F. Ibrahim and N. Touafek, "Max-type system of difference equations with positive two-periodic sequences," Mathematical Methods in the Applied Sciences, vol. 2014, no. 37, pp. 2541-2553, 2014, In press.

[18] A. S. C. Kurbanli and Yalcinkaya, "On the behavior of positive solutions of the system of rational difference equations," Mathematical and Computer Modelling, vol. 53, pp. 12611267, 2011.

[19] A. Y. Özban, "On the system of rational difference equations $x_{n}=a / y_{n}-3, y_{n}=b y_{n}-3 / x_{n}-q y_{n}-q$," Applied Mathematics and Computation, vol. 188, no. 1, pp. 833-837, 2007.

[20] I. Yalcinkaya and Cinar, "Global asymptotic stability of two nonlinear difference equations," Fasciculi Mathematic, vol. 43, pp. 171-180, 2010.

[21] E. A. Grove and G. Ladas, Periodicities in Nonlinear Difference Equations, CRC Press, Boca Raton, FL, USA, 2005.

[22] T. F. Ibrahim and M. A. El-Moneam, "Global stability of a higher-order difference equation," Iranian Journal of Science and Technology, Transactions A: Science, vol. 41, no. 1, pp. 51-58, 2017.

[23] M. T. Aboutaleb, M. A. El-Sayed, and A. E. Hamza, "Stability of the recursive sequence $x_{n}+1=\left(\alpha-\beta x_{n}\right) /\left(\gamma+x_{n}-1\right)$," Journal of Mathematical Analysis and Applications, vol. 261, no. 1, pp. 126-133, 2001.

[24] R. Devault, W. Kosmala, G. Ladas, and Schaultz, "Global behavior of $S_{n+1}=p+S_{n-k} / q S_{n-k}$." Nonlinear Analysis, Theory, Methods and Applications, vol. 47, pp. 83-89, 2004.

[25] E. M. Elabbasy, H. El-Metwally, and E. M. Elsayed, "On the difference equation $x_{n+1}=\alpha x_{n-1}+\beta x_{n-k} / A x_{n-1}+B x_{n-k}$." Acta Mathematica Vietnamica, vol. 33, no. 1, pp. 85-94, 2008.

[26] E. M. Elabbasy, H. El-Metwally, and E. M. Elsayed, "On the Difference equation $x_{n}+1=a x_{n}-b x_{n} /\left(c x_{n}-d x_{n}-1\right)$," $A d$ vances in Difference Equations, vol. 2006, pp. 1-10, 2006.

[27] M. A. El-Moneam and E. M. E. Zayed, "On the rational recursive two sequence $x_{n+1}=a x_{n-k}+\left(b x_{n-k} / c x_{n}+d x_{n-k}\right)$." Acta Mathematica Vietnamica, vol. 35, pp. 355-369, 2010.

[28] E. M. E. Zayad and M. A. El-Moneam, "On the rational recursive sequance $x_{n+1}=\alpha_{0} x_{n}+\alpha_{1} x_{n-2}+\alpha_{2} x_{n-m}+$ $\alpha_{3} x_{n-k} / \beta_{0} x_{n}+\beta_{1} x_{n-2}+\beta_{2} x_{n-m}+\beta_{3} x_{n-k}$." WSEAS Transactions on Mathematics, vol. 11, no. 5, pp. 373-382, 2012. 